\title{
Superparamagnetic relaxation of weakly interacting particles
}

\author{
Mørup, Steen; Tronc, Elisabeth
}

Published in:

Physical Review Letters

Link to article, DOI:

10.1103/PhysRevLett.72.3278

Publication date:

1994

Document Version

Publisher's PDF, also known as Version of record

Link back to DTU Orbit

Citation (APA):

Mørup, S., \& Tronc, E. (1994). Superparamagnetic relaxation of weakly interacting particles. Physical Review Letters, 72(20), 3278-3281. https://doi.org/10.1103/PhysRevLett.72.3278

\section{General rights}

Copyright and moral rights for the publications made accessible in the public portal are retained by the authors and/or other copyright owners and it is a condition of accessing publications that users recognise and abide by the legal requirements associated with these rights.

- Users may download and print one copy of any publication from the public portal for the purpose of private study or research.

- You may not further distribute the material or use it for any profit-making activity or commercial gain

- You may freely distribute the URL identifying the publication in the public portal

If you believe that this document breaches copyright please contact us providing details, and we will remove access to the work immediately and investigate your claim. 


\title{
Superparamagnetic Relaxation of Weakly Interacting Particles
}

\author{
Steen Mфrup \\ Physics Department, Building 307. Technical University of Denmark, DK-2800 Lyngby, Denmark
}

Elisabeth Tronc

Chimie de la Matière Condensée Université Pierre et Marie Curie, 4 Place Jussieu, F-75252 Paris Cedex 05, France

(Received 17 December 1993)

\begin{abstract}
The influence of particle interactions on the superparamagnetic relaxation time has been studied by Mössbauer spectroscopy in samples of maghemite $\left(\gamma-\mathrm{Fe}_{2} \mathrm{O}_{3}\right)$ particles with different particle sizes and particle separations. It is found that the relaxation time decreases with decreasing particle interactions. A new model for the influence of interactions on the superparamagnetic relaxation has been derived and it is shown that this model can explain the observations.
\end{abstract}

PACS numbers: $75.60 . J p, 76.80 .+y$

Ultrafine magnetic particles are of great interest because their properties differ considerably liom those of the corresponding bulk materials [1]. At finite temperatures particles with dimensions below about $10 \mathrm{~nm}$ of ten exhibit superparamagnetic relaxation, i.e., fluctuations of the magnetization vector among the easy directions of magnetization [2]. Superparamagnetic relaxation is currently studied by a number of experimental techniques such as ac and dc susceptibility measurements [3-8], neutron diffraction [9], Mössbauer spectroscopy [6-8,10], ferromagnetic resonance [11], and magnetic force microscopy [12]. Recently the possibility of observing macroscopic quantum tunneling of the magnetization in ultrafine particles has attracted much attention [13].

Ultrafine magnetic particles are commonly found in. for example, soils, rocks, and living organisms, and they have many technological applications, e.g., in magnetic recording media, ferrofluids, and catalysts [1]. The future development of new high-density magnetic recording media containing still smaller magnetic particles may ultimately be limited by superparamagnetic relaxation, which makes the particles unsuitable for the purpose. A new and interesting application of superparamagnetic particles is in materials for magnetic refrigeration well above liquid helium temperature [14]. The magnetic properties of the new nanocrystalline materials, prepared by annealing of metallic glasses, may also be strongly influenced by superparamagnetic relaxation [15]. Thus the subject is of great technological importance.

The superparamagnetic relaxation time is normally expressed by

$$
\tau=\tau_{0} \exp (\Delta E / k T),
$$

where $\tau_{0}$ is of the order of $10^{-10}-10^{-13} \mathrm{~s}[6-8]$ and depends only weakly on temperature. $\Delta E$ is the energy barrier between two easy directions of magnetization, $k$ is Boltzmann's constant, and $T$ is the temperature. For uniaxial particles the magnetic anisotropy energy may be written as $E(\theta)=K V \sin ^{2} \theta$, where $K$ is the magnetic anisotropy energy constant, $V$ is the particle volume, and $\theta$ is the angle between the magnetization vector and an easy direction of magnetization. In this case the energy barrier equals $K V$.

If a small magnetic field $\mathbf{B}$ is applied parallel to the easy direction of a ferromagnetic or ferrimagnetic particle with uniaxial anisotropy the energies at the minima at $\theta=0^{\circ}$ and $\theta=180^{\circ}$ will be different and the transition probabilities for the two opposite transitions will therefore also be different. Introducing $h=\mu B / 2 K V$, where $\mu$ is the magnetic moment of the particle, the two relaxation times can be written [16]

$$
\tau^{ \pm}=\tau_{0}^{ \pm} \exp (\Delta E \pm / k T)
$$

where the energy barriers are given by $\Delta E^{ \pm}=K V(1$ $\pm h)^{2}$ and $\tau_{0}^{ \pm}=\tau_{0}\left(1-h^{2}\right)^{-1}(1 \pm h)^{-1}$. For $h \ll 1$ the exponential gives the most important field dependence of $\tau{ }^{ \pm}$and one may then use the approximation $\tau_{0}^{ \pm} \approx \tau_{0}$.

Equations (1) and (2) were derived for noninteracting particles. In practice, however, magnetic interactions between the particles are often significant and may even result in superferromagnetic ordering at low temperatures, i.e., ordering of the magnetic moments of particles which would be superparamagnetic if they were isolated $[10,17,18]$. The calculation of superparamagnetic relaxation times in real systems of interacting particles is an extremely complex problem, even in the limit of weak interactions. The relaxation times will depend on the detailed geometrical arrangement of the particles and on the orientations of the easy directions of magnetization. Numerical results have been obtained only for the case of two interacting particles with the easy directions parallel to the bond direction of the dipole pair [19]. For manyparticle systems only approximate expressions, based on simple models, have been derived. Shtrikman and Wohlfarth [20] suggested that Eq. (1) should be replaced by the Vogel-Fulcher law, $\tau=\tau_{0} \exp \left[K V / k\left(T-T_{0}\right)\right]$, where $T_{0}$ is a measure of the interaction effect and is proportional to the mean square dipolar field $\left\langle B_{i}{ }^{2}\right\rangle$. Dormann, Bessais, and Fiorani [5] later proposed another model in which they calculated the interaction energy of a particle with each of the neighbor particles separately. The total contribution of interactions to the energy bar- 


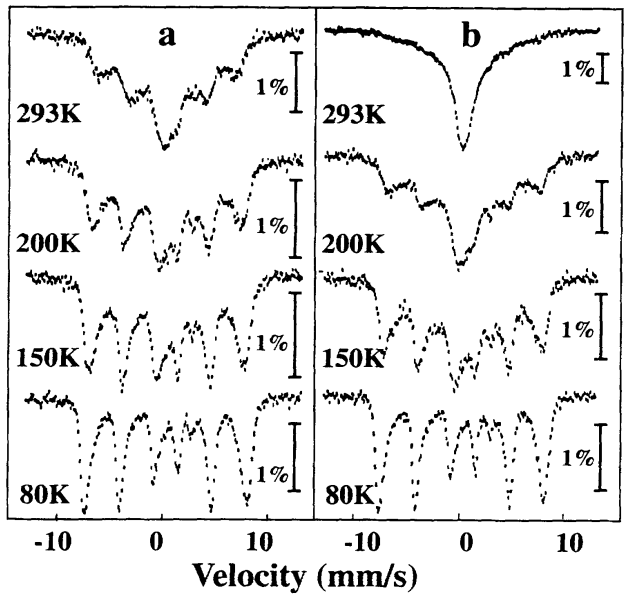

FIG. 1. Mössbauer spectra of $\gamma-\mathrm{Fe}_{2} \mathrm{O}_{3}$ particles dispersed in polyvinylic alcohol. The volume weighted average diameter is $D=8.7 \mathrm{~nm}$ and the interparticle spacing is $5.2 D(\mathrm{a})$ and $1.3 D$ (b).

rier was found by adding the individual contributions. Both of these models predict an increase of the relaxation time with increasing strength of the interactions.

The influence of interactions on the superparamagnetic relaxation has been studied experimentally by use of magnetization measurements [3-5,7] and Mössbauer spectroscopy $[7,10,17,18]$. The blocking temperatures, estimated by the two techniques, have in most cases been found to increase when the average distance between the particles decreases $[3-5,10,17,18]$. This can be explained as freezing of the spins due to interactions $[4,10,17,18]$. Strong interactions are difficult to avoid because most preparation techniques lead to broad particle size distributions and large variations in particle interactions. Experimental studies of the detailed variation of the relaxation time as a function of the strength of the interactions require samples with well-defined interparticle interactions. Recently Prené et al. [7] have developed a preparation technique by which the interactions can be controlled. They varied the $p \mathrm{H}$ of a liquid in which ultrafine maghemite $\left(\gamma-\mathrm{Fe}_{2} \mathrm{O}_{3}\right)$ particles were suspended. In this way the surface electric charge at the particles, which promotes interparticle electrostatic repulsions, could be controlled. Solid samples with minimal aggregation and with different average particle sizes and particle concentrations were prepared by adding a polymer and drying the samples. By using this method a series of samples have been prepared with volume weighted median particle diameters $D$ between 3 and $11 \mathrm{~nm}$ and with average interparticle spacings (center to center) $d=\epsilon D$ with $\epsilon \simeq 5.2$ and 1.3 .

Figure 1 shows Mössbauer spectra of concentrated and dilute samples with $D=8.7 \mathrm{~nm}$ at temperatures between 80 and $293 \mathrm{~K}$. The spectra of both samples show a gradual transition from a six-line (magnetically split) spectrum to a broad singlet and a quadrupole doublet as the

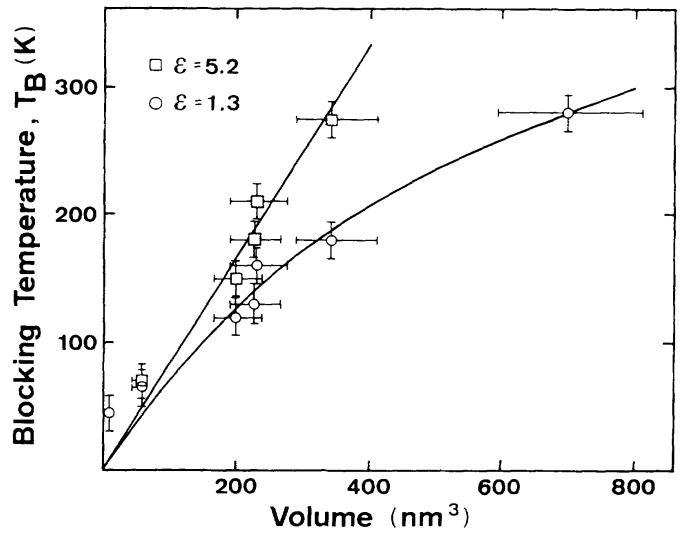

FIG. 2. Blocking temperature, deduced from Mössbauer spectra, as a function of the average particle volume.

temperature is increased. This behavior is typical for spectra of superparamagnetic particles $[8,10,21]$. The blocking temperature $T_{B}$ is defined as the temperature at which the magnetically split and the unsplit components have equal areas. This corresponds to the temperature at which a particle with volume equal to the median of the volume distribution has a relaxation time of the order of the time scale of Mössbauer spectroscopy, $\tau_{m} \approx 5 \times 10^{-9}$ s. It can be seen in Fig. 1 that the blocking temperature is higher for the dilute sample than for the concentrated sample. The same behavior was found for the samples with other median particle diameters. The blocking temperature as a function of the median particle volume is shown in Fig. 2. For all samples the relaxation is faster in the concentrated samples than in the dilute samples. This behavior is opposite to that predicted by the models discussed above. We therefore have reconsidered the influence of interactions on the relaxation time and have developed a new model, which we show is able to account for the experimental results.

We consider a particle $i$ with magnetic moment $\boldsymbol{\mu}$ and with uniaxial magnetic anisotropy. At a given instant of time the particle is exposed to a dipolar field $\mathbf{B}_{i}$, which has contributions from all the surrounding particles. $\mathbf{B}_{i}$ forms and angle $v$, with the easy direction of magnetization, which defines the $z$ direction. The $x$ direction is chosen to be in the plane defined by $\mathbf{B}_{i}$ and the $z$ direction. The angle between the projection of $\boldsymbol{\mu}$ on the $x-y$ plane and the $x$ direction is called $\varphi$. The magnetic energy can now be written

$$
E=K V \sin ^{2} \theta-\mu B_{i}\left(\sin \theta \cos \varphi \sin \iota^{\prime}+\cos \iota \cos \theta\right) .
$$

In the following we assume that $h_{i}=\mu B_{i} / 2 K V \ll 1$. Thus there will be energy minima near $\theta=0^{\circ}$ and $\theta=180^{\circ}$. We have calculated the energy at the minima and the maximum energy at the barrier (near $\theta=90^{\circ}$ ). For a particle at the minimum near $\theta=0^{\circ}$ the energy barrier which must be surmounted in order to reach the minimum near $180^{\circ}$ is given by 


$$
\Delta E(\iota, \varphi) \approx K V\left[1+h_{i}^{2}+2 h_{i}(\cos v-\sin v \cos \varphi)\right] .
$$

Thus for a given value of $v$ the energy barrier is a function of the angle $\varphi$. Therefore, the transition probabilities per unit time, $f(v, \varphi)$, for transitions across the barrier along different paths, corresponding to different values of $\varphi$, are expected to depend on $\varphi$. In the following we assume that the relaxation time for a transition along a given path is given by an expression equivalent to Eq. (1), where $\Delta E$ is replaced by $\Delta E(v, \varphi)$. We also assume that the influence of $\mathbf{B}_{i}$ on $\tau_{0}$ is negligible. The total transition probability per unit time for a particle at $\theta \approx 0$ is then given by

$$
f(v)=\left(2 \pi \tau_{0}\right)^{-1} \int_{0}^{2 \pi} d \varphi \exp \left\{-\frac{\Delta E(v, \varphi)}{k T}\right\} .
$$

For $\beta_{i}=\mu B_{i} / k T \ll 1$ we obtain

$$
\begin{aligned}
f(v)= & \tau_{0}^{-1}\left[1-\beta_{i} \cos v+\left(\beta_{i} / 2\right)^{2}\left(1+\cos ^{2} v\right)\right] \\
& \times \exp \left[-\alpha\left(1+h_{i}^{2}\right)\right],
\end{aligned}
$$

where $\alpha=K V / k T$. The dipole field $\mathbf{B}_{i}$ fluctuates in size and direction. We assume a Gaussian distribution of the values of $\left|\mathbf{B}_{i}\right|$ and that all directions of $\mathbf{B}_{i}$ have the same probability. By integrating over all values of $\mathbf{B}_{i}$ we obtain

$$
\tau \approx \tau_{0} \exp \left[\alpha-\frac{\beta_{\mathrm{av}}^{2}}{3}\left(1-\frac{3}{4} \alpha^{-1}\right)\right]
$$

where $\beta_{\mathrm{av}}^{2}=\mu^{2}\left\langle B_{i}{ }^{2}\right\rangle /(k T)^{2}$.

The basic principles of the model can be summarized as follows: At a given time the particle will be exposed to a dipolar field $\mathbf{B}_{i}$. The magnetization vector fluctuates rapidly in directions close to the energy minimum. These fluctuations (collective magnetic excitations [22,23]) have frequencies of the order of $10^{10}-10^{13} \mathrm{~s}^{-1}$ [23]. Occasionally the magnetization vector will come across the energy barrier at some angle $\varphi$. Averaging over all values of $\varphi$ and $B_{i}$ we obtain the average relaxation time given by Eq. (7). The decrease of the relaxation time due to interactions is related to the lowering of the energy barrier for some values of $\varphi$.

It is now possible to calculate $T_{B}$ in the presence of weak interactions. Noting that $\tau=\tau_{m}$ at $T=T_{B}$ we find from Eq. (7)

$$
T_{B} \approx \frac{K V}{k C}\left[1-h_{\mathrm{av}}^{2}\left(\frac{4}{3} C-1\right)\right],
$$

where $h_{\mathrm{av}}^{2}=\mu^{2}\left\langle B_{i}{ }^{2}\right\rangle /(2 K V)^{2}$ and $C=\ln \left(\tau_{m} / \tau_{0}\right)$. The value of $\left\langle B_{i}^{2}\right\rangle$ at an ion in a paramagnetic material has been calculated by van Vleck [24]. The analogous expression for $\left\langle B_{i}{ }^{2}\right\rangle$ at a particle $i$ in a sample of identical superparamagnetic particles can be written

$$
\left\langle B_{i}{ }^{2}\right\rangle=2\left(\frac{\mu_{0}}{4 \pi}\right)^{2} \mu^{2} \sum_{j} d_{i j}^{-6},
$$

where $\mu_{0}$ is the vacuum permeability and $d_{i j}$ is the distance between the particles $i$ and $j$. Introducing $d_{i j}$ $=a_{i j} \epsilon D$, and assuming that the particles are spherical with identical magnetic moments $\mu=(\pi / 6) D^{3} M$, where $M$ is the saturation magnetization, we obtain

$$
h_{\mathrm{av}}^{2}=\frac{\mu_{0}^{2} M^{4} \epsilon^{-6}}{1152 K^{2}} \sum_{j} a_{i j}^{-6} .
$$

For maghemite particles with dimensions of the order of 5-10 nm, $K \approx(1-4) \times 10^{4} \mathrm{~J} \mathrm{~m}^{-3}[7,25,26]$ and the saturation magnetization of maghemite is $M \approx 4 \times 10^{5}$ A m ${ }^{-1}$. We assume that the value of $\sum_{j} a_{i j}{ }^{-6}$ is independent of particle size and concentration. It may be of the order of 10-20. $\tau_{0}$ is in the range $10^{-10}-10^{-13} \mathrm{~s}$ and thus $C$ is in the range 4-11. Neglecting the width of the size distribution we find from Eqs. (8) and (10) that for $\epsilon=5.2$, the change in $T_{B}$ due to interactions is less than $0.2 \%$ and thus negligible, but for $\epsilon=1.3$ the value of $h_{\text {av }}^{2}\left(\frac{4}{3} C-1\right)$ is in the range $0.2-10$ and therefore the interactions are expected to have a significant influence on $T_{B}$. According to Eqs. (8) and (10) one should expect that the data shown in Fig. 2 can be fitted with straight lines through the origin. However, the best fit of the data for $\epsilon=1.3$ with a straight line has an intercept at about $40 \mathrm{~K}$, and for the smallest particles $T_{B}$ seems to be higher than predicted by the model, both for $\epsilon=1.3$ and $\epsilon=5.2$. There are two obvious explanations for the deviations. First, the value of $K$ is expected to increase with decreasing particle size due to the enhanced influence of surface anisotropy [27]. This can especially explain the relatively high blocking temperatures for the smallest particles. Second, it is well known that spin canting effects reduce the magnetic moments of maghemite particles and the relative amount of canted spins increases with decreasing particle size $[28,29]$. The reduction in the magnetic moment may be described in terms of a nonmagnetic surface layer with a thickness $t \approx 0.3-1.0 \mathrm{~nm} \mathrm{[29].} \mathrm{The} \mathrm{influence}$ of the reduced magnetic moments on $T_{B}$ can be taken into account by replacing $h_{\mathrm{av}}^{2}$ by $h_{\mathrm{av}}^{\prime 2}=h_{\mathrm{av}}^{2}(1-2 t / D)^{12}$. The conditions $h_{\text {av }} \ll 1$ and $\beta_{\text {av }} \ll 1$ used in the derivation of Eq. (8) should then be replaced by $h_{\mathrm{av}}^{\prime} \ll 1$ and $\beta_{\mathrm{av}}^{\prime}=\beta_{\mathrm{av}}(1-2 t / D)^{6} \ll 1$, respectively. The reduction in $T_{B}$ due to the increase in the interactions is less than $35 \%$ (Fig. 2). According to the model this indicates that $h_{\mathrm{av}}^{\prime 2}\left(\frac{4}{3} C-1\right)<0.35$, i.e., $h_{\mathrm{av}}^{\prime 2}<0.08$ and $\beta_{\mathrm{av}}^{\prime 2} \leqslant 1$ at $T$ $\gtrsim T_{B}$ even for the smallest possible value of $C$. The approximations used for the derivation of Eq. (8) therefore seem reasonable.

The fits of the data points shown in Fig. 2 were obtained using the model described above. For $\epsilon=5.2$ the fit is a straight line from the slope of which we find that $K / k C=0.81 \mathrm{~K} \mathrm{~nm}^{-3}$. The data points for $\epsilon=1.3$ can be fitted well with a range of combinations of the parameters $h_{\mathrm{av}}^{2}\left(\frac{4}{3} C-1\right)$ and $t$. The curve shown is the best fit calculated with $K / k C=0.81 \mathrm{~K} \mathrm{~nm}^{-3}, h_{\mathrm{av}}^{2}\left(\frac{4}{3} C-1\right)=2.13$, and $2 t=1.24 \mathrm{~nm}$. The fit shows that the model is able to ac- 
count for all the results, except those for the smallest particles which are expected to be most influenced by an increase in $K$ due to surface effects [27].

It is thus concluded that the present model for the influence of weak interactions on the superparamagnetic relaxation can explain the observed dependence of the blocking temperature on the strength of the interaction.

We finally emphasize that the model is only valid for small values of $\beta_{\mathrm{av}}^{\prime}$. At low temperatures $\left(\beta_{\mathrm{av}}^{\prime} \gtrsim 1\right)$, the magnetic interaction will result in freezing of the magnetic moments into a spin-glass-like state [4]. In the frozen states with lowest energy the direction of $\mathbf{B}_{i}$ will be close to the easy direction of magnetization for a large fraction of the particles such that the energy barriers for reversal of the magnetization of these particles increase, resulting in longer relaxation times. For the concentrated samples $B_{\mathrm{av}}^{\prime} \approx 1$ at temperatures of the order of $100 \mathrm{~K}$. Studies of the initial susceptibility have shown maxima at temperatures $T_{\max }<100 \mathrm{~K}$ and the values of $T_{\max }$ were found to increase with increasing concentration [7]. This behavior is opposite to that predicted by the model discussed above, but it can be explained by the freezing of the magnetic moments due to interaction in the concentrated samples at low temperatures [4].

Chimie de la Matière Condensée is CNRS URA No. 1466.

[1] See, for example, Magnetic Properties of Fine Particles, edited by J. L. Dormann and D. Fiorani (North-Holland, Amsterdam, 1992).

[2] L. Néel, Ann. Geophys. 5, 99 (1949).

[3] M. El-Hilo, K. O’Grady, and R. W. Chantrell, J. Magn. Magn. Mater. 114, 295 (1992); 114, 307 (1992); 117, 21 (1992).

[4] W. Luo et al., Phys. Rev. Lett. 67, 2721 (1991).

[5] J. L. Dormann, L. Bessais, and D. Fiorani, J. Phys. C 21, 2015 (1988).

[6] C. Johansson et al., J. Magn. Magn. Mater. 122, 125
(1993); S. Linderoth et al., ibid., 124, 269 (1993).

[7] P. Prené et al., IEEE Trans. Magn. 29, 2658 (1993).

[8] D. P. E. Dickson et al., J. Magn. Magn. Mater. 125, 325 (1993).

[9] I. Mirebeau et al., J. Magn. Magn. Mater. 104-107, 1560 (1992); M. Hennion et al., in Magnetic Properties of Fine Particles (Ref. [1]), p. 27.

[10] S. Mørup, Hyperfine Interact. 60, 959 (1990).

[11] H. M. Ziethen, A. X. Trautwein, and H. Winkler, Hyperfine Interact. 56, 1733 (1990).

[12] M. Lederman, G. A. Gibson, and S. Schultz, J. Appl. Phys. 73, 6961 (1993).

[13] E. M. Chudnovsky and L. Gunther, Phys. Rev. Lett. 60 , 661 (1988); Phys. Rev. B 37, 9455 (1988); E. M. Chudnovsky, J. Appl. Phys. 73, 6697 (1993); B. Barbara et al., ibid. 73, 6703 (1993); J. Tejada, X. X. Zhang, and L. I. Balcells, ibid. 73, 6709 (1993).

[14] R. D. McMichael et al., J. Magn. Magn. Mater. 111, 29 (1992); L. H. Bennett et al., J. Appl. Phys. 73, 6507 (1993); R. D. Shull et al., in Magnetic Properties of Fine Particles (Ref. [1]), p. 161.

[15] A. Slawska-Waniewska et al., Phys. Rev. B 46, 14594 (1992).

[16] W. F. Brown, Jr., Phys. Rev. 130, 1677 (1963).

[17] S. Mørup et al., J. Magn. Magn. Mater. 40, 163 (1983).

[18] P. V. Hendriksen et al., Nucl. Instrum. Methods Phys. Res., Sect. B 76, 138 (1993).

[19] A. Lyberatos and R. W. Chantrell, J. Appl. Phys. 73, 6501 (1993).

[20] S. Shtrikman and E. P. Wohlfarth, Phys. Lett. 85A, 467 (1981).

[21] W. Kündig et al., Phys. Rev. 142, 377 (1966).

[22] S. Mørup and H. Topsoe, Appl. Phys. 11, 63 (1976).

[23] S. Mørup, J. Magn. Magn. Mater. 37, 39 (1983).

[24] J. H. van Vleck, J. Chem. Phys. 5, 370 (1937).

[25] P. Prené et al., Hyperfine Interact. (to be published).

[26] P. V. Hendriksen et al., J. Phys. Condens. Matter (to be published).

[27] F. Bødker, S. Mørup, and S. Linderoth, Phys. Rev. Lett. 72, 282 (1994).

[28] J. M. D. Coey, Phys. Rev. Lett. 27, 1140 (1971).

[29] A. H. Morrish and K. Haneda, J. Magn. Magn. Mater. 35, 105 (1983). 